\title{
1 The Bayesian Superorganism: externalised memories facilitate distributed sampling
}

2 Edmund R. Hunt ${ }^{1}$, Nigel R. Franks ${ }^{1}$, Roland J. Baddeley ${ }^{2}$

31 School of Biological Sciences, University of Bristol, Life Sciences Building, 24 Tyndall Avenue, Bristol, BS8 1TQ, UK

42 2School of Experimental Psychology, University of Bristol, 12a Priory Road, Bristol, BS8 1TU, UK

5 ORCIDS: ERH, 0000-0002-9647-124X; NRF, 0000-0001-8139-9604; RJB, 0000-0002-7431-6580

6 Authors for correspondence: email: edmund.hunt@bristol.ac.uk, roland.baddeley@bristol.ac.uk 7

\section{Abstract}

9 A key challenge for any animal (or sampling technique) is to avoid wasting time by searching for

10 resources (information) in places already found to be unprofitable. In biology, this challenge is

11 particularly strong when the organism is a central place forager - returning to a nest between foraging

12 bouts - because it is destined repeatedly to cover much the same ground. This problem will be

13 particularly acute if many individuals forage from the same central place, as in social insects such as the

14 ants. Foraging (sampling) performance may be greatly enhanced by coordinating movement trajectories

15 such that each ant ('walker') visits separate parts of the surrounding (unknown) space. We find experimental evidence for an externalised spatial memory in Temnothorax albipennis ants: chemical markers (either pheromones or cues such as cuticular hydrocarbon footprints) that are used by nestmates to mark explored space. We show these markers could be used by the ants to scout the space surrounding their nest more efficiently through indirect coordination. We also develop a simple model of

20 this marking behaviour that can be applied in the context of Markov chain Monte Carlo methods

21 (Baddeley et al. 2019). This substantially enhances the performance of standard methods like the Metropolis-Hastings algorithm in sampling from sparse probability distributions (such as those confronted by the ants) with little additional computational cost. Our Bayesian framework for superorganismal behaviour motivates the evolution of exploratory mechanisms such as trail marking in terms of enhanced collective information processing. 


\section{Introduction}

30 Exploring an unfamiliar, changing environment in search of valuable resources such as food or potential

31 nest sites is a challenge for many organisms. A memory of where one has already explored, to avoid

32 revisiting unprofitable locations, would generally seem to be an advantage. A spatial memory of foraging

33 locations, for example, is likely to be beneficial but it would entail physiological costs. These include the

34 metabolic overhead of a bigger brain (memory storage capacity) and the cost of encoding and retrieving memories (brain activity); these costs have to be traded-off against the benefit of improved foraging performance [1]. One way to circumvent the cost of carrying memories internally is to store the information externally in the environment. Indeed, such externalised information storage may have been the historical precursor to the development of internalised memory storage and retrieval $[2,3]$. Use of external markers as memory has been demonstrated in arguably simple organisms, including the slime mould Physarum polycephalum [4]. Markers may be left in the environment to signify the presence or absence of good foraging or nest-making prospects at that location, so that when an animal returns it can make an appropriate and timely decision about the expenditure of its efforts.

An analogous problem to animals searching for food sources in an unfamiliar environment is that of sampling efficiently from unknown probability distributions. Markov chain Monte Carlo (MCMC) methods have been developed to generate such samples but suffer from the same problem of potentially revisiting the same area of probability space repeatedly. Although methods such as the MetropolisHastings algorithm show random walk behaviour in their sampling trajectory, they are still popular techniques because of their ease of implementation and computational simplicity. As discussed in the first paper of our series on the 'Bayesian superorganism' [5], MCMC methods can be used as a model of animal movement that enacts the optimal probability matching strategy for collective foraging ('gambling') [6]. There, we drew a parallel with advances in efficiency in MCMC methods, to more

52 adaptive animal behaviours emerging in natural history: for example, from the random-walk type

53 behaviour of the Metropolis-Hastings algorithm $[7,8]$ to the correlated random walks produced by

54 Hamiltonian Monte Carlo [9]. The performance boost provided by externalised memories, discussed here, 
may similarly be understood as an evolutionary advancement in collective information processing capabilities.

the footprints of nestmates during the reconnaissance of an unfamiliar space, and that this enhances the efficiency of the colony-level collective exploration; (2) we develop a bio-inspired trail avoidance method for the purpose of Markov chain Monte Carlo sampling that similarly uses a memory of where the walker has visited to obtain a representative sample more rapidly. This method is easy to implement and computationally simple, and offers significant improvements in performance for the sort of sparse distributions that ants and other organisms also have to sample from routinely. In the next section we introduce some more biological background on chemical markers (externalised memories), but the reader familiar with this topic may proceed to the Methods section.

66

\section{Further biological background on chemical markers}

In addition to expediting the process of environmental search, external markers can also communicate one's presence to friendly or competitor conspecifics. Some animals are well-known for marking territory: for instance wolves (Canis lupus) use scent marks to maintain their territories [10] while mice (Mus musculus) use olfactory cues to help establish their territorial boundaries [11]. In the Hymenoptera, the honeybee Apis mellifera ligustica marks visited flowers with a scent and rejects flowers that have been recently visited; it responds more strongly to its own markers than that of its nestmates [12]. Such repellent scent-marks should help the bee to forage more efficiently. As in the ants, these marks may simply be hydrocarbon footprints rather than costly signals [13]. Ants are known to engage in two distinct approaches to marking the area around their nest. In territorial marking, ants will defend the marked area against intra- and inter-specific intruders. By contrast, home-range markers label areas that the colony knows to be hospitable and available for foraging, but such areas are not defended against other ant colonies [14]. Major workers of the African weaver ant Oecophylla longinoda mark their territories with persistent pheromones that are distinguishable to the ants at the colony level [15], whereas workers of the leafcutter ant Atta cephalotes deposit 'nest exit pheromones' in the vicinity of 
their nest entrances, helping to orient workers and hence enhancing the efficiency of leaf gathering [16].

Lasius niger ants have been found to engage in home-range marking, which rather than territorial marking may help them to enlarge their potential foraging space alongside other colonies, in a 'shared information' strategy [17].

influence the behaviour of other ants, ants have also been shown to respond to cues such as the cuticular hydrocarbon footprints left by other ants. These can also be used to distinguish between different colonies [18], and potentially also to recognize different castes, as in a study of Reticulitermes termites [19]. They can also be detected by ant predators, and so this deposition is not without costs [20]. The response ants make to detecting such markers depends on whether the cue is from a nestmate, a competing colony, or a different species altogether, because depending on the relationship between sender and receiver, it could represent a threat or useful information about the location of foraging resources [21]. The role of the ant species (dominant or submissive) in the local community can be key in this respect [22].

In our study organism, the house-hunting rock ant $T$. albipennis, there is evidence for pheromone use in navigating to a new nest site [23,24] as well as in T. curvispinosus [25]. Other Temnothorax species are also known to use individual-specific trail pheromones for orientation outside their nests: $T$. affinis [26] and T. unifasciatus [27]. T. albipennis is thought to use an individual-specific pheromone trail during the nest-site assessment process: to measure the floor area using the 'Buffon's needle' algorithm, an ant experiences a lower crossing rate of its own trail when the nest is larger. If its trail was not individualspecific only one ant could do this before the trail was confused with those of other scouting nestmates

103 [28]. T. albipennis colonies are also found to avoid conspecific colonies when choosing where to locate its 104 nest, as a result of odour cues left by foreign colonies [29]. Pheromones are also probably used by the 105 ants when scouting potential new nest sites, since when previously assessed nest sites are replaced with 106 clean nest sites, colonies are more likely to pick the poor option, having lost their reconnaissance

107 information [30]. T. rugatulus was found to use an alarm pheromone that elicits two different behaviours 
avoid danger; while when released near the ants' home nest it attracts them to defend against a threat

110 [31].

111 Chemical marks left by harvester ants (Pogonomyrmex) during exploration for new food have

112 been suggested as a way of avoiding redundant repeat searches in that area [32,33]. The usefulness of an

113 avoidance behaviour during the searching phase for food was commented upon by Nonacs in a study of

114 Lasius pallitarsis ants. Such a behaviour was predicted to be more helpful for solitary foragers, than for

115 species with mass recruitment to prey items that need teams of workers to carry them home.

116 Experimental investigation found that the ants show a state-dependent avoidance of the path of previous

117 nestmates [34]. This question has recently been revisited when evidence was found that Myrmica rubra

118 (Myrmicinae) ants avoid the footprints of their nestmates, which suggests that they are using the

119 information it conveys to inform their foraging or scouting decisions [22]. However, this behaviour was

120 not observed in the four other ant species in the study: Formica polyctena (Formicinae), Formica

121 rufibarbis (Formicinae), Lasius niger (Formicinae), and Tetramorium caespitum (Myrmicinae). T.

122 albipennis is also in the subfamily Myrmicinae, and we predicted that it too uses cuticular hydrocarbon

123 footprints left by previous exploring nestmates to scout an unknown area surrounding its nest more

124 efficiently. Here, we present evidence that $T$. albipennis indeed avoids the footprints of nestmates during

125 the reconnaissance of an unfamiliar space, and for the first time show that it enhances the efficiency of

126 the colony-level collective exploration.

\section{Data processing and analysis}

130 We examine data from a previous study of $T$. albipennis ant movement patterns $[35,36]$. These data

131 present two experimental regimes, one in which the foraging arena was entirely novel to exploring ants,

132 because it was cleaned by the experimenter in advance, and one in which previous traces of the ants'

133 activities remained. We call these treatments 'No cleaning' (NC) and 'Cleaning' (C). For both treatments,

134 we used 18 ants, 6 from each of the three colonies, for a total of 36 ants. Treatments were alternated

135 over six consecutive days. The colonies inhabited small artificial nests with a $2 \mathrm{~mm}$ diameter entrance 
136 hole drilled into the centre of the top. A colony was placed into a $90 \times 90 \mathrm{~cm}$ arena and a $15 \times 15 \mathrm{~cm}$ white

137 paper mask with a $1 \times 1 \mathrm{~cm}$ central hole affixed over the top, with a further $1.5 \times 1.5 \mathrm{~cm}$ white paper

138 removable cover to block the nest entrance. This created a smooth surface (with a raised area over the

139 nest) to allow continuous tracking of a single ant. The paper cover was removed to allow a single ant out

140 of the nest, and the entrance cover was then replaced, so that the ant would explore freely in isolation

141 for 45 minutes before being removed to a separate dish for the duration of the experiment. The

142 exploration trajectory of the ant was recorded by a camera mounted on a motorised gantry system,

143 which followed the ant's movements and recorded its path as a sequence of $(x, y)$ points spaced by 0.1

144 seconds. In the NC treatment, six ants were consecutively released from the same colony on the same

145 day, and pheromone signals and/or other cues such as cuticular hydrocarbon footprints were allowed to

146 accumulate. In the $\mathrm{C}$ treatment, the arena surface was cleaned before the subsequent ant was allowed

147 into the arena, to prevent chemical communication between successive ants. See the 'Methodology and

148 apparatus' section of Hunt et al. [36] for a more detailed description of methods and Basari et al. [37] for

149 a photo and more details on the gantry system.

150 In the previous study [36] the focus of the analysis was on the characteristics of movement

151 'events' - a period of non-zero speed followed by stopping - and the effect of treatments on metrics such

152 as average speed and the correlation between the average speed of consecutive movement events. Here,

153 we examine the effect of the treatment on exploration behaviour - how efficiently the ants reconnoitre

154 an unfamiliar experimental arena - with the hypothesis that the ants use chemical information from

155 previous exploring nestmates to do this more quickly, by avoiding moving through regions of the space

156 that have already been somehow marked as explored. We test this hypothesis of enhanced exploration

157 efficiency in the following way. In nature the ants might benefit from sampling preferentially from

158 surrounding regions of higher quality, in the sense of being more likely to contain valuable resources such

159 as food [5] or a potential new nest site [38]; and would want to spend less time in 'empty' regions of the

160 space that contain little of relevance to their reproductive fitness. High-quality regions are likely to be

161 associated with cues that help the ants navigate toward them: for instance, a good potential nest site 
163 from warmer ambient temperatures. Therefore, in a natural environment we would not expect the ants

164 to explore uniformly in the region around their nest; and while we might naively suppose that an empty

165 experimental arena represents a uniform environment, from an ant's perspective it may be somewhat

166 heterogeneous (lighting, air currents, vibrations, magnetic field, temperature, thigmotaxis around the

167 edges of the paper mask with a focus on the corners, and so forth), despite an experimenter's best

168 efforts. Therefore, rather than measure the ants' exploration efficiency by reference to how quickly they

169 fill uniform space, we take the 'target distribution' that ants are trying to sample from as being

170 approximated by the actual exploratory trajectories taken by all 36 ants. While this combines data from

171 two different treatments, with social information presumably present in one ('no cleaning'), this larger

172 sample is more likely to approximate the ants' exploration preferences. Although this 'target distribution'

173 may seem an unconventional approach, it will account for any heterogeneity in terms of the lighting

174 environment, for example (though best efforts were taken to control conditions), and also differential

175 responses in terms of lateralized behaviour. For instance, a preference for viewing landmarks with the

176 right eye has been suggested [37], which may affect exploration behaviour, and also a leftward turning

177 bias when exploring nest sites [39], which may relate to these ants having slightly asymmetric eyes [40].

178 We create a target distribution for the exploring ants in the following way, using MATLAB 2015b.

179 The exploration trajectories for all 36 ants are combined (a total exploration time of 27 hours), and trajectory points located inside the central region where the $15 \times 15 \mathrm{~cm}$ paper mask is located are removed, because many of the ants spend a significant proportion of their time familiarising themselves with their immediate surroundings and engaging in thigmotaxis (edge following) around the mask before

183 beginning reconnaissance bouts away from the central region. The $(x, y)$ coordinates were then

184 transformed from a Cartesian to a log-polar system $(\theta, \ln r)$, where $-\pi \leq \theta \leq \pi$ and $4.3<\ln r<6.5$

185 (the approximate size of the arena in $\mathrm{mm}$ excluding the central area). This transformation of trajectories

186 makes the distribution of visits to different parts of the arena more uniform, because a spatial histogram

187 effectively has larger bins for more radially distant areas, and smaller bins for the more densely visited

188 central region containing the nest. This makes statistical tests for differences between trajectories more

189 powerful. A periodic boundary for angle $\theta$ was created by copying the points in the rectangular region 
$190 \theta \in[-\pi, 0], \ln r \in[4.3,6.5]$ into a new region $[\pi, 2 \pi]$ and similarly copying $[0, \pi]$ into $[-2 \pi,-\pi]$ to

191 create a larger space $-2 \pi \leq \theta \leq 2 \pi$. This periodic boundary prevented 'ringing' effects at the edge

192 when a gaussian blur was applied over all the points in the space, to create a continuous distribution. This

193 also functions effectively as data interpolation for regions without data, because the small ants ( $1 \mathrm{~mm}$

194 wide) will not visit every point in the $900 \mathrm{~mm}$ square arena. The coordinates from all 36 trajectories were

195 counted using a 2D histogram on a grid $1257 \times 881$ ( $\theta$ step size $0.01, \ln r$ step size 0.0025$)$. This

196 represents where in the arena the ants tend to spend their time exploring. A gaussian blur has a

197 parameter $\sigma$ which controls the width of the blur. Since the ants are small (only around $1 \mathrm{~mm}$ wide) a

198 fairly wide blur is necessary to transform their exploration trajectories into a continuous distribution. The

$199 \sigma$ used was set equal to 30, which corresponds to larger blurs for more radially distant (less visited)

200 regions when applied in $\ln r$ space: roughly $6 \mathrm{~mm}$ wide closest to the nest and $40 \mathrm{~mm}$ wide toward the

201 edge of the arena. After the blur was applied to the 2D histogram, the distribution was then truncated

202 back to its original size such that $-\pi \leq \theta \leq \pi$ again, and normalised such that the sum of all the points

203 was equal to 1 , as in a discrete probability distribution where the value at each arena grid position

204 represents the probability of an ant visiting it. The ants' exploration trajectories and resultant empirical

205 target distribution is shown in Figure 1, and individual and cumulative trajectories are shown in Figures S3-6. 

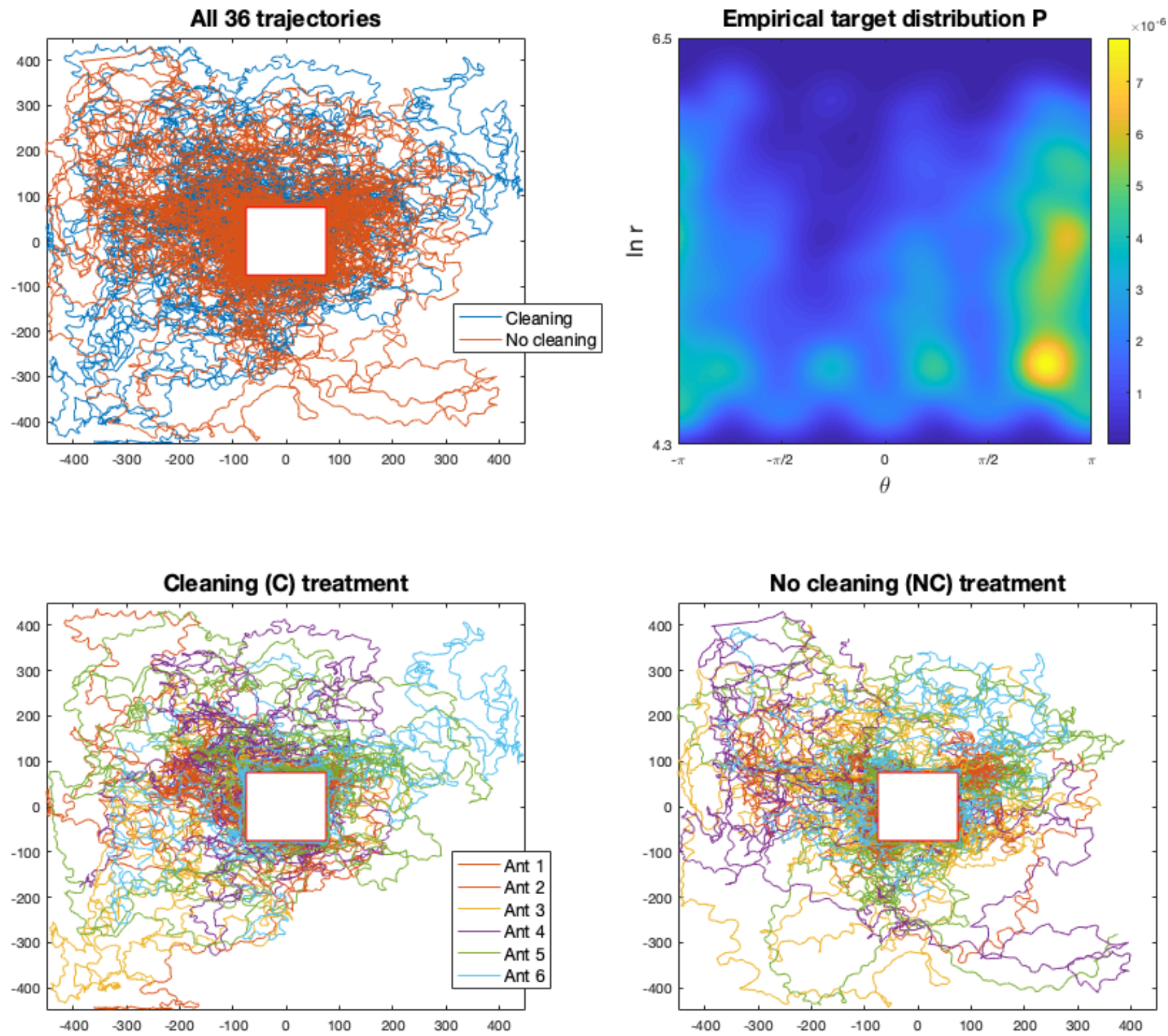

Figure 1. The ants' exploration trajectories for the two treatments and empirical target distribution P. All

208 trajectories are shown in the top left pane, marked by treatment, while trajectories are shown by

209 treatment and ant order in the bottom panes ( 3 ants for each order in the sequence).

As shown in Figure 1, the ants in the cleaning treatment made a somewhat uneven exploration

211 of the arena, spending more time in the top-left (quadrant 2) and not visiting the bottom-right corner of

212 the space (quadrant 4), in contrast to the ants in the no cleaning treatment. We sought to compare our

213 data with an independent study of a different ant species to investigate whether such exploration

214 heterogeneity had precedent elsewhere. We examined the data of Khuong et al. [41], which recorded the

215 exploration behaviour of Lasius niger ants placed individually in an empty arena of various inclines. Their

216 treatment with zero inclination recorded the movement of 69 ants from the centre of a $50 \times 50 \mathrm{~cm}$ arena

217 to the edge, whereupon the ant was removed. It should be noted that these worker ants are larger at 
around 3-5mm long compared to $2 \mathrm{~mm}$ Temnothorax ants, and so our arena was larger from the ants'

219 perspective as well as in absolute terms. We examine which side of the arena the L. niger ants exited the

$22050 \times 50 \mathrm{~cm}$ region and replicate this analysis for our own ants (Figure S1). Although the exploration of the

221 ants was uniform in the centre of the arena, it had a bimodal angular distribution as the radius of

222 exploration increased (Figure S2). We perform a Chi-Square goodness of fit test for a uniform distribution

223 of exit sides (i.e. an expectation of $25 \%$ on each side), and an exact multinomial test using the $\mathrm{R}$ package

224 'EMT' [42] for our smaller number of observed exit points. Because the first ants in the NC treatment experience a clean arena, we switch these three ants to the $\mathrm{C}$ grouping for the purpose of this analysis. trajectories were combined by colony from all ants up to ant $1,2, \ldots 6$ out of the nest. For instance, to measure the progress by ant 2 of the NC treatment, the trajectories of 2 ants were combined (the first ant, and the second) to see its cumulative progress toward the goal. The process described above was

230 then repeated to obtain a probability distribution, which was then compared against the final distribution

231 using the cross-entropy (CE) as a measure of their similarity, where a lower CE signifies greater progress

232 toward the equilibrium distribution of a well-explored arena (see also [5]). In the last step the three

233 colony-level CE measures were averaged to obtain a mean cross-entropy for the NC treatment by ant 2.

234 Our hypothesis that the ants use chemical information from preceding nestmates to explore more efficiently would correspond to a CE that falls faster in the NC treatment than the C treatment.

237 information from nestmates, it is also quite possible that individual ants make use of their own

238 pheromone markers or other cues left behind, like their own footprints, to avoid wasting time revisiting

239 regions of space they have already walked through. To assess the benefit of individual-level memory

240 (both internal and external), we constructed simulated trajectories by randomly sampling from a vector

241 of the movements of all 36 ants. These 'Markov' ants provided a memoryless benchmark of exploration

242 performance, as ants that do not avoid their own trails, because there is neither externalised memory

243 (chemical markers) nor internal memory (in their brain) of where they have been. A trajectory of 45

244 minutes of exploration was constructed by concatenating 1 second periods of movement that stayed 
within the arena boundary (a 90×90 cm space). The performance of 100 'colonies' of these Markov ants (6 per colony, as with the real colonies) was compared in the same way.

To assess the statistical significance of one treatment converging more quickly on the final

248 distribution than the other, a permutation test was used, on ants 2-6 out of the nest. The first ant is not

249 included in the test for statistical significance since it is expected that the two treatments are equivalent

250 at this stage: the first ant explores an arena empty of any chemical information. A non-parametric test is

251 appropriate since the distribution of the CE for each treatment is unknown, and relatively few data points are being compared (15 from each treatment). A permutation test was also used to assess whether the average distance travelled by each exploring ant is different in the NC and C treatments.

\section{Trail avoidance model}

256 In previous research $[5,38]$ we have proposed that a central challenge that an ant colony confronts, that

257 of trying to identify regions of value in an unknown surrounding environment, is analogous to the

258 problem found in statistics, physics, etc. of efficiently sampling from complex probability distributions or

259 computing numerical integrations. This is because in order to sample from a probability distribution

260 efficiently, one must identify regions of high probability density, but these are not known a priori but only

261 after evaluating the probability density function at those locations. Similarly, an ant needs to find regions of high quality, but does not know where they are unless it expends effort (time, energy) in exploration $[5,38]$. An ant colony has a key advantage over a solitary animal: it is composed of many workers, which can cooperate to benefit the colony as a whole, working together as one 'superorganism' [43]. Each worker could set off in a different direction to that of its nestmates, and hence all of the surrounding area could be quickly explored. However, the ants lack any central controller to direct their movements so as

267 not to overlap and waste time exploring the same region several times; instead they must make their

268 decisions about where to move based only on locally available information [44]. A key feature of the

269 Markov chain Monte Carlo (MCMC) movement models we have proposed is the use of local quality

270 gradient information in deciding where to move next: it would seem reasonable to expect ants (and other 
considered is that this gradient may be modified by the ants themselves, by making use of their sophisticated chemical communication systems [14]. We suggest that ants may leave behind chemical markers, either deliberately (i.e. pheromones) or incidentally (such as hydrocarbons from their footprints), that convey information about where they and their nestmates have already explored. Such markers may be thought of as 'negative', in the sense that an ant seeking to explore the environment will seek to avoid them, and as reducing the quality gradient in that direction, such that other alternative directions have a relatively higher gradient. ant trying to sample from an unknown resource quality distribution $P$ also leaves behind a consistent marker at every step that it takes, which corresponds to its subjective experience or model of the world $M$ (but which does not require any internal memory in the ant itself). $M$ starts off as a uniform prior of the world (e.g. a distribution of ones for each location) but then is added to (e.g. +1 ) at each visited location. This is equivalent to Bayesian updating with a Dirichlet prior, because this is the conjugate to the multinomial distribution [45]. $M$ can be renormalised at each time step to be a probability distribution that sums to 1 . Over time, as the ant more thoroughly explores the distribution $P$, this model will converge $M \rightarrow P$, with more markers accumulating in regions of higher probability. Since the objective of the ant is to sample from regions of high probability first, one way to prioritise such regions is to sample from $P^{2}$, since this will substantially increase the relative value of high probability regions over low probability regions. For instance, an ant would rather visit a region of 0.2 probability over a region of 0.1 , with the former being of 2 times the quality as the latter. If this is squared, this becomes a relative

292 value of $\frac{0.2^{2}}{0.1^{2}}=\frac{0.04}{0.01}=4$ times higher, making this region of $\mathrm{P}$ space much more attractive, with a sharper 293 gradient toward it too. Therefore, an effective objective for ant exploration (or indeed MCMC methods) is 294 to sample from:

$$
P \times \frac{P}{M}
$$
which converges in time to sample from $P$ as $P / M \rightarrow 1$, but which should sample from high probability regions first as a combination of the $P^{2}$ (attractive) and $M$ (repulsive) terms. Such an objective function 
applied in conjunction with the most basic approaches such as Metropolis-Hastings, which are computationally simple. A 'pseudocode' representation of our approach is shown in Table 1.

301

302

303

304

305

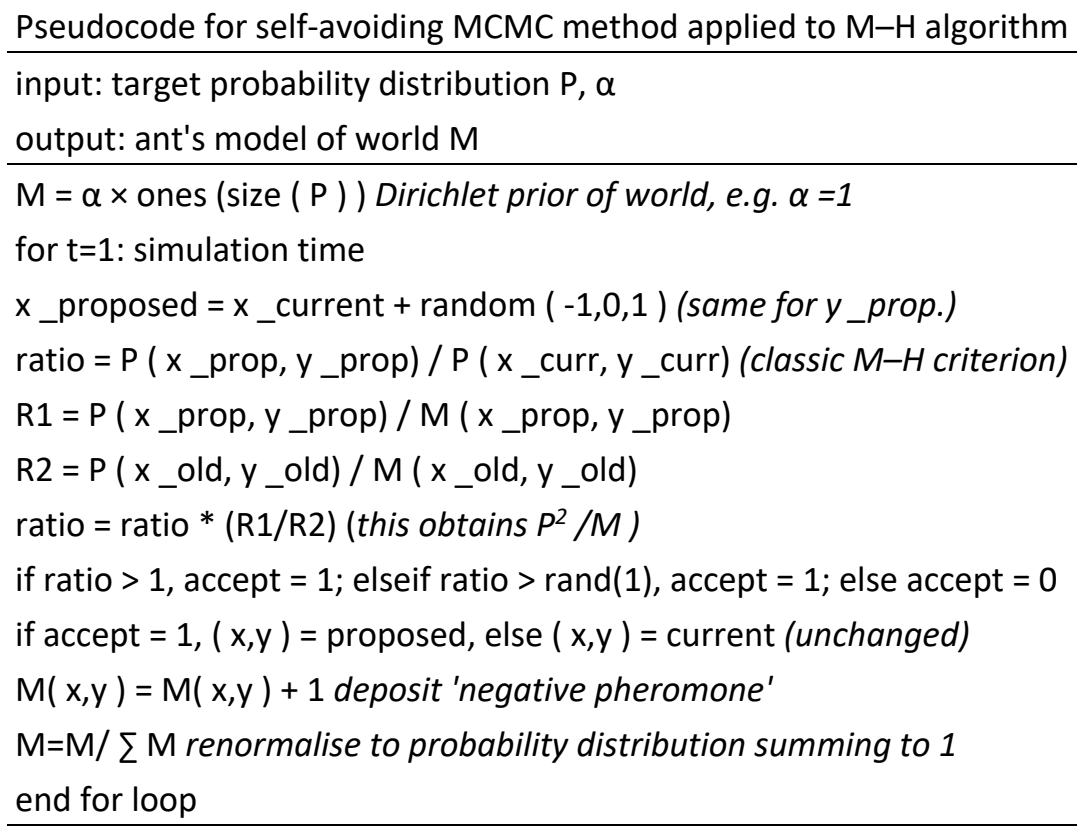

Simulations of this 'trail-MCMC' model of ant exploration (or sampling approach) are presented in the results, using a Metropolis-Hastings $(\mathrm{M}-\mathrm{H})$ method to sample from both a random distribution (corresponding to a noisy, sparse world) and the empirical distribution of all the ant trajectories. The random distribution (shown in Figure 4) was Dirichlet-distributed, generated by sampling from a gamma distribution of shape $k$ and scale parameter $\theta=1$. This results in mostly low-probability cells with a few higher-probability cells, which corresponds to the ants' ecology where most space is unsuitable for locating the colony's nest.

The $\mathrm{M}-\mathrm{H}$ method samples from the distributions using periodic boundary conditions. To assess the effect of cleaning (that is, removing the externalised spatial memory of the ants) the simulations are also run under a 'cleaning' condition where the memory is reset five times, to correspond to the five cleaning bouts in the experimental study (after each ant has explored, for six ants). To ease this comparison the simulations are run for $\mathrm{T}=60,000$ time steps, or 10,000 time steps per 'ant'. The performance is compared to a $\mathrm{M}-\mathrm{H}$ method sampling from the standard objective distribution $P$ by 
318 examining the rate of fall in cross-entropy between the sample and the target $P$ (as previously discussed

319 in [5]).

320

$321 \quad$ Results

\section{Ant exploration trajectories}

323 The rate of convergence of the exploring ants toward the final distribution is shown in Figure 2; the ants

324 in the no cleaning treatment converge significantly more quickly than those in the cleaning treatment

$325(p=0.0161$, permutation test on ants $2-6$ across the 3 colonies. The analysis was repeated for Gaussian

326 blur size $\sigma=20$, resulting in $\mathrm{p}=0.0394$, and $\sigma=40$, resulting in $\mathrm{p}=0.0098$ ). This is despite the average

327 distance travelled by the ants from each treatment not being significantly different. The average distance

328 is $18.58 \pm 0.77 \mathrm{~m}$ (s.e. of the mean) in the NC treatment and $17.57 \pm 0.77 \mathrm{~m}$ in the $\mathrm{C}$ treatment $(\mathrm{p}=0.21$,

329 permutation test). As expected, without any memory (externalised or internal) the simulated Markov

330 ants converge on the target more slowly than either of the experimental treatments, because they are

331 essentially engaged in a random walk around the arena.

332 As anticipated, the ants were not uniform in how they explored the arena. Ants in both

333 treatments tended to spend more time in the second quadrant of the arena and less time in quadrant 4

334 (Figure 1); this could represent a tendency in the ants to respond to unknown environmental gradients,

335 or some kind of intrinsic behavioural tendency. Ants in the no cleaning treatment tended to cover more

336 of the arena as a whole (Figure 1). We confirmed that heterogeneity in exploration patterns is not unique

337 to our data by examining the Lasius niger data of Khuong et al. [41] which showed a significant tendency

338 for ants to leave through two of the four virtual arena sides $\left(\chi_{3}^{2}=39.46, p=1.384 \times 10^{-8}\right.$, Figure $\left.S 1\right)$. While

339 there was a significant exit bias for ants in our cleaning treatment (exact multinomial test: $p=0.0137$ ), this

340 tendency was not found to be significant in the no cleaning treatment (EMT: $p=0.1102$ ). 


\section{Faster convergence on final distribution $\mathbf{P}$ without cleaning}

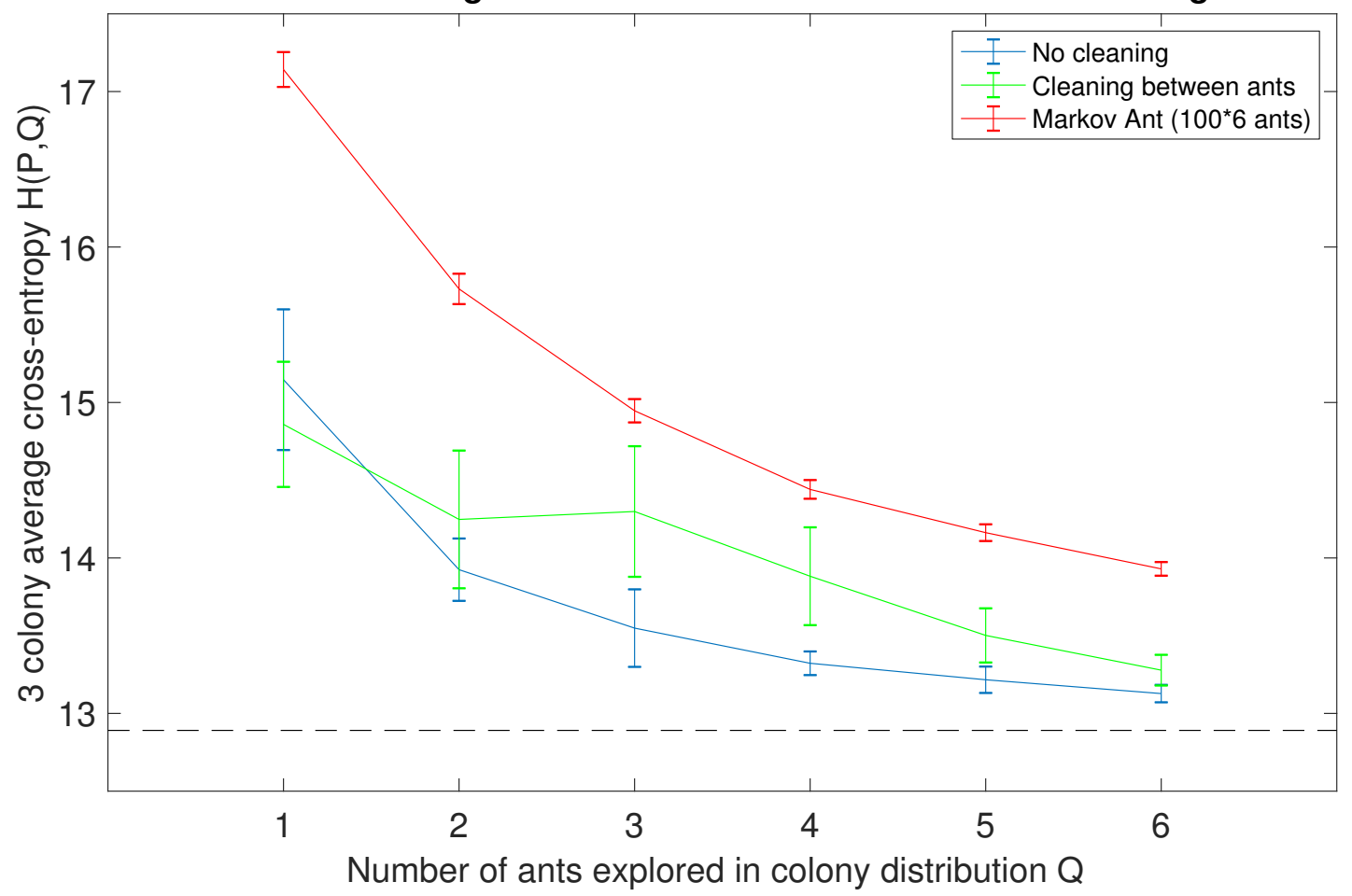

343 Figure 2. The ants in the no cleaning treatment converge most quickly toward the final distribution, 344 indicating that they benefit from chemical information left by preceding nest mates. The error bars indicate one standard error of the mean; a permutation test on ants 2-6 indicate that the cross-entropy is significantly lower in NC than C ( $p=0.0161)$. The simulated 'Markov ant' converges slowest, because it benefits neither from the chemical markers (externalised spatial memory) nor internal memory about where it has already visited. The cross-entropy minimum is indicated by the dashed black line.

\section{Trail avoidance model simulations}

352 An example simulation of the models (standard $\mathrm{M}-\mathrm{H}$, trail-avoidance $\mathrm{M}-\mathrm{H}$ with and without cleaning) for

353 the sparse distribution is shown in Figure 3. Such a distribution corresponds to a region of empty space where the ants would wish to avoid sampling from the same parts of the distribution repeatedly. It might be thought of as a 'zoomed-in' patch of empty space in the ants' environment, as opposed to the more patchy empirical distribution in Figure 4. Figure 3 shows that the standard $\mathrm{M}-\mathrm{H}$ method converges slowly to the target distribution when all the space needs to be sampled from roughly equally: with a random walk considerable time is wasted going over previous ground. By contrast, the trail-avoidance $\mathrm{M}-\mathrm{H}$ methods converge much more quickly, as seen in a more rapidly falling cross-entropy. In a way similar to

360 the experimental result shown in Figure 2, the advantage of not cleaning the pheromone trail is not

361 immediately apparent but becomes evident by 'ant $3^{\prime}$ ( $t=3 \times 10^{4}$ on the $x$-axis), because more 

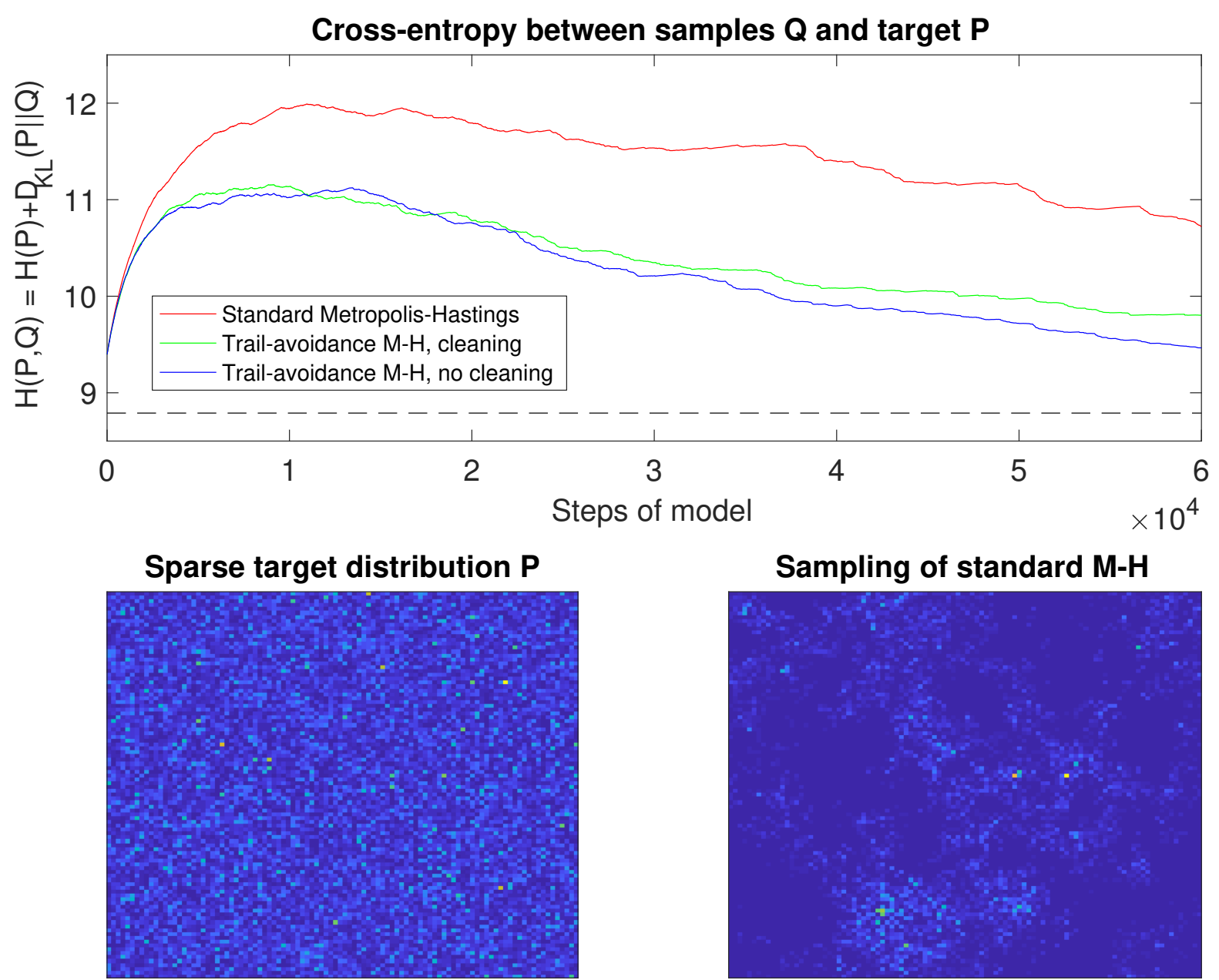

Sampling of standard $\mathrm{M}-\mathrm{H}$

Trail avoidance $\mathrm{M}-\mathrm{H}$ Cleaning 5 times (6 'ants')

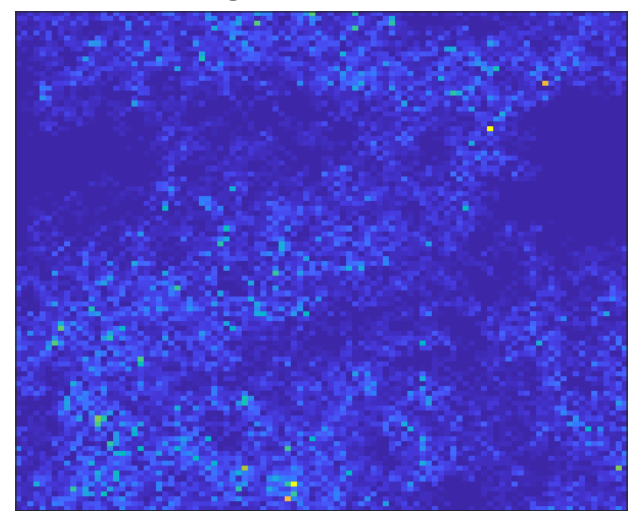

Trail avoidance $\mathbf{M}-\mathrm{H}$ No cleaning

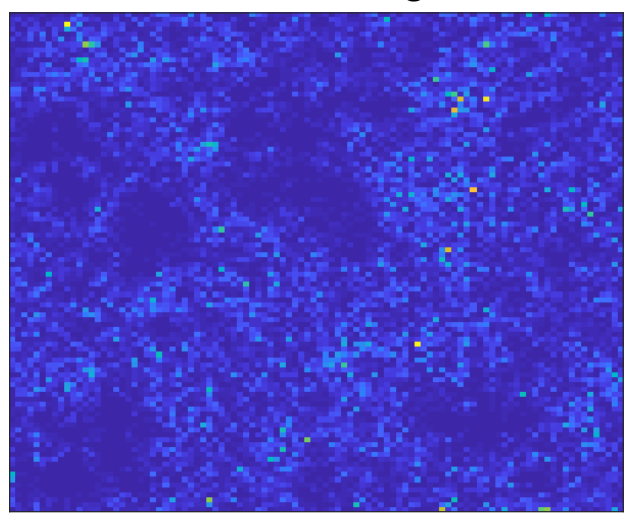

Figure 3. The trail avoidance model converges much more quickly to the simulated sparse target distribution, while 'cleaning' (removing the externalised memory of the sampling trajectory) slows its progress somewhat. The target (cross-entropy minimum) is shown by the dashed black line. 
369 The result of a set of simulations sampling from the empirical distribution of Figure 1 is shown in Figure 4.

370 This does not show such a large improvement in performance for trail-avoidance M-H over standard M-

$371 \mathrm{H}$, because significant portions of the probability mass are more readily accessible to random walk-like

372 behaviour. Nevertheless, the standard $\mathrm{M}-\mathrm{H}$ method is seen to have spent more time in the quadrant 2

373 patch, in keeping with the general problem the ants also face of prematurely favouring a local resource,

374 rather than exploring relevant information in the global distribution. In comparison, the no cleaning trail-

375 avoidance $\mathrm{M}-\mathrm{H}$ samples from the whole distribution in proportion to their quality in this example, and

376 converges most quickly to the target distribution as a result. The cleaning simulation shows intermediate

377 performance, sampling more effectively than $\mathrm{M}-\mathrm{H}$ but not exploring the whole distribution as thoroughly

378 as when the pheromone memory is allowed to accumulate. 


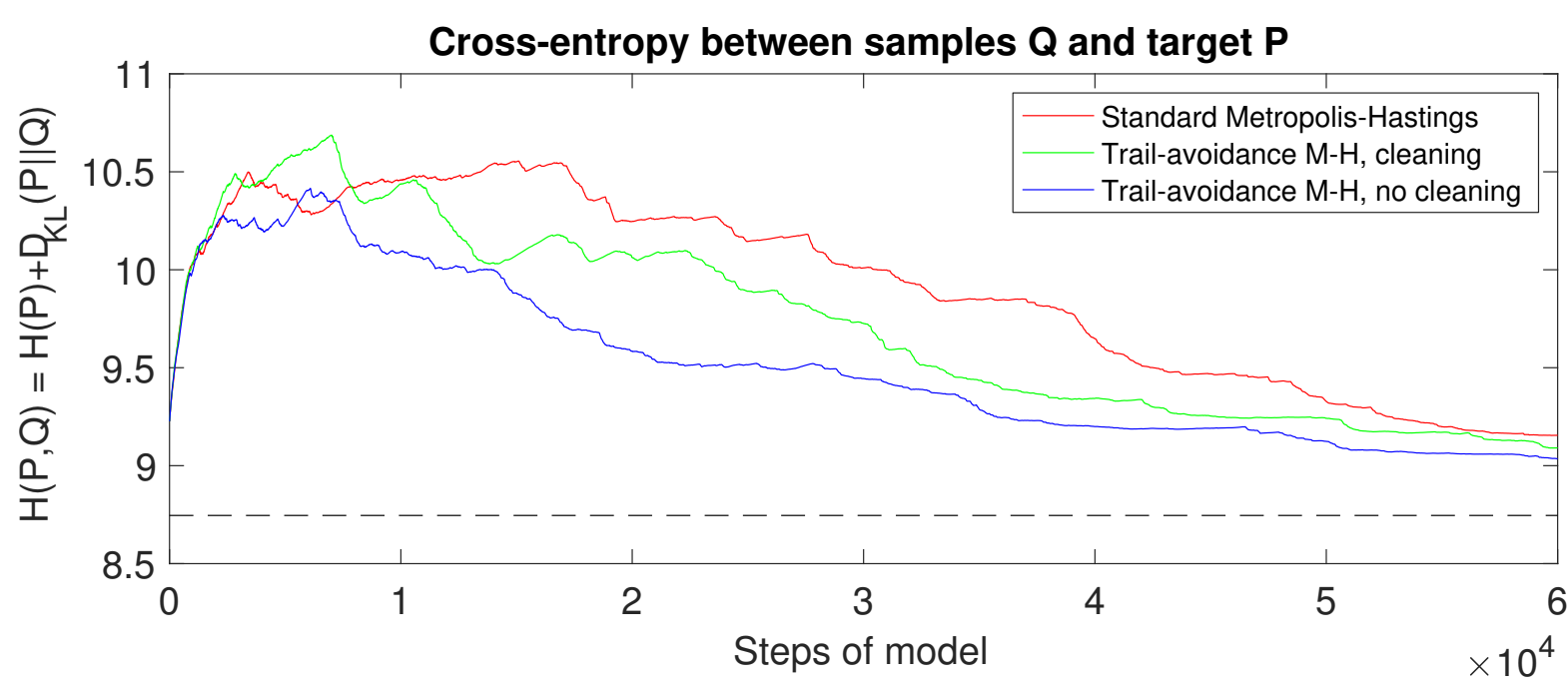

\section{Empirical target distribution $\mathbf{P}$}

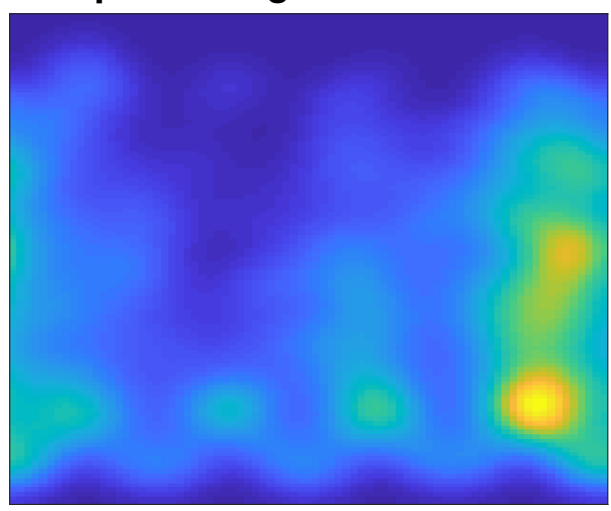

Sampling of standard $\mathrm{M}-\mathrm{H}$

Figure 4. The trail avoidance model again converges more quickly to the target. The $\mathrm{M}-\mathrm{H}$ model is more likely to get 'stuck' in higher probability regions and take longer to get to other important parts of the distribution. However, the performance gain is less obvious than in the preceding gamma distribution example. 
Ants and other organisms confront the challenge of having to explore unknown environments while avoiding wasting time and incurring other costs and risks, which would occur if there are repeated visits to the same unprofitable areas. One strategy to avoid time-wasting would be for the animal to

391 maintain an internal memory of where it has been, by using techniques such as learning the configuration

392 of local landmarks (allothetic navigation) [46], or counting steps in a certain direction from the nest (path

393 integration, or other idiothetic mechanisms such as optic flow). However, the ants are pre-eminent manipulators of terrestrial chemical information [14]. Analysis of experimental data [35] presented here provides initial evidence that Temnothorax albipennis ants use chemical depositions - either pheromones or cues such as cuticular hydrocarbon footprints - to explore an unknown arena more efficiently. This is because the ants in the treatment where chemical information from nestmates is allowed to accumulate converge on the colony's target distribution more quickly than when that information is systematically cleaned away. Such indirect coordination between ants is consistent with previous analysis of this data, which found that the correlation between the speeds of successive movements was significantly lower in the 'no cleaning' treatment ( 0.234 vs. a correlation of 0.407 in the 'cleaning' treatment), which we suggested is because ants are responding to social information by changing their movement patterns

403 [36]. Such colony-level indications of a potential negative marker could be confirmed in future research by using coupled gas chromatography/mass spectrometry to identify candidate marker compounds from arena swabs in the 'no cleaning' treatment. The influence of such candidate compounds could then be tested experimentally on individuals or colonies, as in Sasaki et al., for example [31].

408 Myrmica rubra, that found evidence for nestmate footprint avoidance though did not demonstrate 409 enhanced search efficiency [22]. The avoidance behaviour is seen to be such that the trajectories of two 410 successive ants will nevertheless intersect several times: persistent avoidance would, in effect, paint an

411 ant into a corner. Instead the suggestion of a previous ant's reconnaissance leads to a reweighting of 412 exploration toward a different region of space. In practice, where an ant holds private information about 413 a source of food, for instance, that conflicts with social information (an exploratory marker) it is likely to 
dominate the decision about whether to return to the space when it is held with high confidence

415 (probability) [56]. When there is a coherence between private memory and social information, this may

416 help to boost ants' 'confidence' in their own memories and walk more directly (straighter and faster)

417 toward their goal [57].

418 The results shown in Figure 2 indicate the value of both internal and external forms of memory:

419 the 'Markov ants' have neither, while the ants in the two treatments (cleaning and no cleaning) have

420 both available, but the no cleaning ants to the fullest extent. It may be possible to assess the relative

contribution to exploration efficiency from own versus others' pheromones by covering an exploring

ant's gaster with paint [54], while the use of visual landmarks to navigate [46] can similarly be prevented

by covering an ant's eyes [55]. It may be that ants benefit primarily from their own chemical marks and only secondarily from nestmate marks. These experimental possibilities are summarised in Table 2 , and could be used to quantify the value of different information sources for exploration, in informational terms, using the cross-entropy method introduced here.

Table 2. Additional conditions that could be investigated to assess the value for exploration of different information sources.

\begin{tabular}{|c|c|c|c|c|}
\hline \multirow{3}{*}{ Condition } & \multicolumn{2}{|c|}{ Internal Memory } & \multirow{2}{*}{\multicolumn{2}{|c|}{$\begin{array}{c}\text { External Memory } \\
\text { Pheromones }\end{array}$}} \\
\hline & \multirow{2}{*}{$\begin{array}{c}\text { Other } \\
\text { (odometry etc.) }\end{array}$} & \multirow{2}{*}{ Visual } & & \\
\hline & & & Own & Others \\
\hline Markov ant & $\mathrm{X}$ & $\mathrm{X}$ & $\mathrm{X}$ & $\mathrm{X}$ \\
\hline Covered eyes & $\checkmark$ & $\mathrm{x}$ & $\checkmark$ & $\checkmark$ \\
\hline $\begin{array}{l}\text { Covered } \\
\text { gaster + } \\
\text { cleaning }\end{array}$ & $\checkmark$ & $\checkmark$ & $\mathrm{x}$ & $\mathrm{X}$ \\
\hline $\begin{array}{l}\text { Covered } \\
\text { gaster }\end{array}$ & $\checkmark$ & $\checkmark$ & $\mathrm{X}$ & $\checkmark$ \\
\hline Cleaning & $\checkmark$ & $\checkmark$ & $\checkmark$ & $\mathrm{X}$ \\
\hline No cleaning & $\checkmark$ & $\checkmark$ & $\checkmark$ & $\checkmark$ \\
\hline
\end{tabular}

431 quadrant 4; this was less evident in the no cleaning treatment where the arena was not cleaned between

432 exploration bouts (Figures 1, S1). Seeking to understand this unexplained tendency, we compared our trajectories with a study of Lasius niger by Khuong et al. [41]. They reported the following: "Regarding the statistics of exit heading direction, we observed a visible excess in favor of the lower right part of the canvas... We have no explanation for this bias so far, and it calls for further examination and testing." 
436 Their methodology does not specify whether there was arena cleaning between trials, and so it is

437 possible that cuticular hydrocarbons influenced movement $[17,47]$. If this possibility can be excluded,

438 Khuong et al. noted that some eusocial insects are sensitive to the magnetic field [48-51], and the

439 movement bias in our data is in roughly the same south-westerly direction (Figure S1), though

440 magnetoreception in Temnothorax seems improbable. A priori, one may have an expectation of a

441 correlated random walk in a homogeneous environment, resulting in diffusive behaviour and a

442 homogeneous exploration density at the macroscopic scale (e.g. [52]). What may be observed in Figures

443 S1 and S2 is a switch between behaviours from intensive search in the central starting point (e.g. for the

444 nest entrance in our data) to a stereotyped search (e.g. [53]). at which point the influence of a subtle

445 environmental gradient, possibly interacting with intrinsic tendencies with respect to lateralisation,

446 become evident in displacement direction. As with an MCMC method, given enough time, one would still

447 expect all parts of the arena to be explored. Further review and testing across ant species should confirm

448 whether heterogeneous exploration densities observed here are idiosyncratic or more widespread than

449 previously recognised.

The experimental evidence for path avoidance of nestmates helped to inspire a new approach to

451 tackling an analogous problem in the world of MCMC methods: slow convergence times owing to repeated sampling of the same region of probability space. The original MCMC method, MetropolisHastings, is well known for its random walk behaviour, yet is still a popular method because of its ease of implementation. We have shown that its efficiency can be substantially enhanced if it samples from the distribution $P \times P / M$, where $M$ is a model (distribution) of where the MCMC walker has visited in the space, rather than simply $P$ (the target distribution). The performance gain is particularly noticeable when sampling from sparse distributions, where the need to avoid revisiting previous parts of the space is especially acute. T. albipennis ants live in just such environments - where most of their surroundings hold little of relevance in terms of fitness, either food or potential nest sites - and hence such a

460 mechanism should indeed be favoured by evolution. A shared substrate may serve effectively as a

461 collective memory store, boosting the information-processing capability of the colony beyond the

462 bottleneck of a single ant's memory. Here we have just examined the enhancement to sampling 
463 performance for a single walker (ant) - of course in real colonies there will be many simultaneous walkers

464 (ants), and their individual trajectories will be non-independent. More sophisticated MCMC methods that

465 deploy independent parallel walkers, e.g. that presented by Wang and Landau [58], can be compared to

466 our approach.

467 The model presented here retains a perpetual memory of where the ant has walked, although

468 ongoing movement and pheromone deposition will lessen the relative weighting of past trails as

469 pheromone accumulates elsewhere. In reality, pheromones will decay in strength over time. Although

470 this is unavoidable, in a dynamic foraging environment it may well be preferable that repellent markers

471 decay over time in any event, because they may quickly lose their relevance and even be maladaptive if

472 they inhibit an ant from returning to an area where food has become available. Similar considerations

473 may apply as with animals deciding how to weigh past observational memories with current ones; an

474 exponential weighting is suitable in a Bayesian analysis [59]. It would be straightforward to introduce a

475 reduction to the potency of the markers over time by multiplying the model by a decay factor, as in

$476 e^{-\lambda t} M$, where $\lambda$ is the decay constant. Theoretical consideration may be given to the optimal decay

477 constant for different environments (or probability distributions), with sparse environments with a lower

478 chance of opportunistic (randomly appearing) food favouring a lower constant, i.e. a slower decay. Such

479 predictions may be readily tested in different ant species associated with different ecologies.

predicted the value of inhibitory (negative) signals, before their empirical confirmation [60]. In certain

483 signal has been found in Pharaoh's ants Monomorium pharaonis to mark the unrewarding branch on a

484 trail bifurcation. Concentrated at decision points - where the path branches - it could be used to

485 complement attractive trail pheromones or to prevent an attractive pheromone becoming too strong

486 through runaway positive feedback [62]. These ants are thought to have at least three types of foraging

487 trail pheromone: one long-lasting attractive pheromone, thought to mark out the overall foraging

488 network, and two short-lived attractive and repellent pheromones, to mark out short term food

489 locations. The repellent pheromone was found to last more than twice as long as the short-lived 
490 attractive pheromone [63]. Simulations indicate that repellent pheromone increases the robustness and

491 flexibility of the colony's overall foraging response [64]. The usefulness of such negative or repellent

492 markers has also been demonstrated in a swarm robotics context, in simulation $[65,66]$, digitally on the

493 robots themselves [67,68], or with real-world light-based markers [69-71]. Just as theoretical models of

494 self-organisation predicted the value of negative markers before they were empirically confirmed, it is

495 possible that advancements in Markov chain Chain Monte methods may inadvertently explain, or pre-

496 empt the discovery of, certain behavioural mechanisms. Similarly, the biology of collective exploration is

497 potentially a rich source of bio-inspiration for MCMC.

498 


\section{Conclusion}

500 Many organisms face the challenge of identifying and exploiting food sources in an unfamiliar and

501 changing environment. A common pitfall in the search process is revisiting previously explored regions of

502 space that do not contain anything of value. An evolutionarily ancient way of avoiding this problem is to

503 leave an externalised 'memory' of the previous visit by depositing a chemical marker that can be used to

504 make a quick decision not to re-enter that space when it is encountered later. For superorganisms like

505 ants, that seek to maximise their foraging performance at the level of the colony, pheromone signals or

506 cues such as cuticular hydrocarbon footprints may be used to coordinate the movement decisions of

507 their nestmates (i.e. through stigmergy) such that an unfamiliar space is quickly explored. Here, we

508 presented evidence for avoidance of the exploration of previous nestmates in Temnothorax albipennis

509 ants, solitary foragers who would indeed particularly benefit from such a behaviour. We also

510 demonstrated that a path avoidance behaviour enhances the exploration efficiency of the ants at the

511 colony level.

512 In addition to empirical findings, after noting the analogy between ant exploration and sampling

513 from unknown probability distributions, we developed an ant-inspired enhancement to Markov chain

514 Monte Carlo methods, whereby a memory is kept of where a walker has moved through the probability

515 space. Such a method was shown to significantly enhance the efficiency of the Metropolis-Hastings

516 method $[7,8]$ when sampling from sparse probability distributions, because the random walk type

517 behaviour of the method is reduced, but at little computational cost in comparison to more advanced

518 momentum-based methods such as Hamiltonian Monte Carlo [9]. When the 'superorganism' [43] is

519 examined using our Bayesian framework $[5,38]$, behavioural mechanisms such as individual trail markers

520 become better understood in an ultimate sense [72] in relation to their facilitation of adaptive collective

521 information-processing capabilities. 
523 Data accessibility. The Temnothorax albipennis ant data analysed here can be found at:

524 http://dx.doi.org/10.5061/dryad.jk53j

525 The Lasius niger data analysed here is associated with [41] and can be found at:

526 https://doi.org/10.1371/journal.pone.0076531.s005

527 Author contributions. ERH drafted the paper, ran the simulations and analysed the data; NRF advised on 528 social insect biology; RJB originated and advised on the theoretical concepts. All authors contributed to 529 the present draft.

530 Funding statement. E.R.H. thanks the UK Engineering and Physical Sciences Research Council (grants no.

531 EP/I013717/1 to the Bristol Centre for Complexity Sciences and EP/N509619/1, DTP 2016-17

532 University of Bristol, EPSRC DTP Doctoral Prize).

533 Ethics statement. The ant Temnothorax albipennis is not subject to any licencing regime for use in

534 experiments. The ants were humanely cared for throughout the experiment.

535 Competing interests. We have no competing interests.

539 1. Fagan WF et al. 2013 Spatial memory and animal movement. Ecol. Lett. 16, 1316-1329.

540 2. Chung JR, Kwon J, Choe Y. 2009 Evolution of recollection and prediction in neural networks. Proc. Int.

\section{References} Jt. Conf. Neural Networks, 571-577. (doi:10.1109/IJCNN.2009.5179065)

3. Chung JR, Choe Y. 2009 Emergence of Memory-like Behavior in Reactive Agents Using External Markers. In 2009 21st IEEE International Conference on Tools with Artificial Intelligence, pp. 404-408. (doi:10.1109/ICTAI.2009.116)

4. Reid CR, Latty T, Dussutour A, Beekman M. 2012 Slime mold uses an externalized spatial 'memory' to navigate in complex environments. Proc. Natl. Acad. Sci. 109, 17490-17494. (doi:10.1073/pnas.1215037109)

5. Baddeley RJ, Franks NR, Hunt ER. 2019 Optimal foraging and the information theory of gambling. J. R. Soc. Interface 16, 20190162. (doi:10.1098/rsif.2019.0162)

6. Kelly JL. 1956 A new interpretation of information rate. Bell Syst. Tech. J. 35, 917-926.

7. Metropolis N, Rosenbluth AW, Rosenbluth MN, Teller AH, Teller E. 1953 Equation of state calculations by fast computing machines. J. Chem. Phys. 21, 1087-1092. (doi:10.1063/1.1699114)

8. Hastings WK. 1970 Monte Carlo sampling methods using Markov chains and their applications. Biometrika 57, 97-. (doi:10.2307/2334940)

9. Duane S, Kennedy AD, Pendleton BJ, Roweth D. 1987 Hybrid Monte Carlo. Phys. Lett. B 195, 216-222. (doi:10.1016/0370-2693(87)91197-x)

10. Peters RP, Mech LD. 1975 Scent-Marking in Wolves. Am. Sci. 63, 628-637. (doi:http://www.jstor.org/stable/27845779)

11. Mackintosh JH. 1973 Factors affecting the recognition of territory boundaries by mice (Mus musculus). Anim. Behav. 21, 464-470. (doi:10.1016/S0003-3472(73)80006-5)

12. Giurfa M. 1993 The repellent scent-mark of the honeybee Apis mellifera tigustica and its role as communication cue during foraging. Insectes Soc. 40, 59-67. (doi:10.1007/bf01338832)

13. Eltz T. 2006 Tracing pollinator footprints on natural flowers. J. Chem. Ecol. 32, 907-915. (doi:10.1007/s10886-006-9055-6)

14. Hölldobler B, Wilson EO. 1990 The Ants. Harvard University Press.

15. Hölldobler B, Wilson EO. 1977 Colony-specific territorial pheromone in the African weaver ant Oecophylla longinoda (Latreille). Proc. Natl. Acad. Sci. 74, 2072-2075.

16. Hölldobler B, Wilson EO. 1986 Nest area exploration and recognition in leafcutter ants (Atta cephalotes). J. Insect Physiol. 32, 143-150. (doi:10.1016/0022-1910(86)90133-2)

17. Devigne C, Detrain C. 2002 Collective exploration and area marking in the ant Lasius niger. Insectes Soc. 49, 357-362. (doi:10.1007/pl00012659)

18. Akino T, Yamaoka R. 2005 Trail discrimination signal of Lasius japonicus (Hymenoptera: Formicidae). 
19. Bagnères $A-G$, Rivière $G$, Clément J-L. 1998 Artificial neural network modeling of caste odor discrimination based on cuticular hydrocarbons in termites. Chemoecology 8, 201-209. (doi:10.1007/s000490050026)

20. Cárdenas M, Jiroš P, Pekár S. 2012 Selective olfactory attention of a specialised predator to intraspecific chemical signals of its prey. Naturwissenschaften 99, 597-605. (doi:10.1007/s00114-0120938-9)

21. Wüst M, Menzel F. 2016 I smell where you walked - how chemical cues influence movement decisions in ants. Oikos 126. (doi:10.1111/oik.03332)

22. Binz H, Foitzik S, Staab F, Menzel F. 2014 The chemistry of competition: Exploitation of heterospecific cues depends on the dominance rank in the community. Anim. Behav. 94, 45-53.

(doi:10.1016/j.anbehav.2014.05.024)

23. Pratt SC, Brooks SE, Franks NR. 2001 The Use of Edges in Visual Navigation by the Ant Leptothorax albipennis. Ethology 107, 1125-1136. (doi:10.1046/j.1439-0310.2001.00749.x)

24. McLeman MA, Pratt SC, Franks NR. 2002 Navigation using visual landmarks by the ant Leptothorax albipennis. Insectes Soc. 49, 203-208. (doi:10.1007/s00040-002-8302-2)

25. Bowens SR, Glatt DP, Pratt SC. 2013 Visual Navigation during Colony Emigration by the Ant Temnothorax curvispinosus. PLoS One 8. (doi:10.1371/journal.pone.0064367)

26. Maschwitz U, Lenz S, Buschinger A. 1986 Individual specific trails in the ant Leptothorax affinis (Formicidae: Myrmicinae). Experientia 42, 1173-1174. (doi:10.1007/bf01941298)

27. Aron S, Deneubourg JL, Pasteels JM. 1988 Visual cues and trail-following idiosyncrasy in Leptothorax unifasciatus: An orientation process during foraging. Insectes Soc. 35, 355-366.

(doi:10.1007/bf02225811)

28. Mallon EB, Franks NR. 2000 Ants estimate area using Buffon's needle. Proc. R. Soc. B Biol. Sci. 267, 765-770. (doi:10.1098/rspb.2000.1069)

29. Franks NR, Dornhaus A, Hitchcock G, Guillem R, Hooper J, Webb C. 2007 Avoidance of conspecific colonies during nest choice by ants. Anim. Behav. 73, 525-534. (doi:10.1016/j.anbehav.2006.05.020)

30. Franks NR, Hooper JW, Dornhaus A, Aukett PJ, Hayward AL, Berghoff SM. 2007 Reconnaissance and latent learning in ants. Proc. R. Soc. London B Biol. Sci. 274, 1505-1509. (doi:10.1098/rspb.2007.0138)

31. Sasaki T, Hölldobler B, Millar JG, Pratt SC. 2014 A context-dependent alarm signal in the ant Temnothorax rugatulus. J. Exp. Biol. 217, 3229-3236. (doi:10.1242/jeb.106849)

32. Holldobler B, Wilson EO. 1970 Recruitment Trails in the Harvester Ant Pogonomyrmex Badius. Psyche (Stuttg). 77, 385-399. (doi:10.1155/1970/38470)

33. Carroll CR, Janzen DH. 1973 Ecology of Foraging by Ants. Annu. Rev. Ecol. Syst. 4, 231-257. (doi:10.1146/annurev.es.04.110173.001311)

34. Nonacs P. 1991 Exploratory behavior of Lasius pallitarsis ants encountering novel areas. Insectes Soc. 38, 345-349. (doi:10.1007/BF01241870)

35. Hunt ER, Baddeley RJ, Worley A, Sendova-Franks AB, Franks NR. 2016 Data from: Ants determine their next move at rest: motor planning and causality in complex systems. R. Soc. Open Sci. 3, 150534.

(doi:10.1098/rsos.150534)

36. Hunt ER, Baddeley RJ, Worley A, Sendova-Franks AB, Franks NR. 2016 Ants determine their next move at rest: motor planning and causality in complex systems. R. Soc. Open Sci. 3, 150534.

(doi:10.1098/rsos.150534)

37. Basari N, Bruendl AC, Hemingway CE, Roberts NW, Sendova-Franks AB, Franks NR. 2014 Landmarks and ant search strategies after interrupted tandem runs. J. Exp. Biol. 217, 944-954.

(doi:10.1242/jeb.087296)

38. Hunt ER, Franks NR, Baddeley RJ. 2018 The Bayesian Superorganism I: collective probability estimation. bioRxiv , 468942. (doi:10.1101/468942)

39. Hunt ER, O'Shea-Wheller T, Albery GF, Bridger TH, Gumn M, Franks NR. 2014 Ants show a leftward turning bias when exploring unknown nest sites. Biol. Lett. 10, 20140945. (doi:10.1098/rsbl.2014.0945)

40. Hunt ER, Dornan C, Sendova-Franks AB, Franks NR. 2018 Asymmetric ommatidia count and behavioural lateralization in the ant Temnothorax albipennis. Sci. Rep. 8. (doi:10.1038/s41598-018-23652-4)

41. Khuong A, Lecheval V, Fournier R, Blanco S, Weitz S, Bezian JJ, Gautrais J. 2013 How do ants make sense of gravity? A Boltzmann Walker analysis of Lasius niger trajectories on various inclines. PLoS One 8. (doi:10.1371/journal.pone.0076531)

42. Menzel U. 2013 Exact Multinomial Test: Goodness-of-Fit Test for Discrete Multivariate Data. $R$ Packag. version 1.1. 
630 43. Hölldobler B, Wilson EO. 2009 The superorganism: the beauty, elegance, and strangeness of insect

631

632

633

634

635

636

637

638

639

640

641

642

643

644

645

646

647

648

649

650

651

652

653

654

655

656

657

658

659

660

661

662

663

664

665

666

667

668

669

670

671

672

673

674

675

676

677

678

679

680

681

682

683

684

685

686 societies. WW Norton \& Company.

44. Camazine S, Deneubourg J-L, Franks NR, Sneyd J, Theraulaz G, Bonabeau E. 2001 Self-Organization in Biological Systems. Princeton, NJ: Princeton University Press.

45. Frigyik BA, Kapila A, Gupta MR. 2010 Introduction to the Dirichlet Distribution and Related Processes. Department of Electrical Engineering, University of Washington.

46. Hunt ER, Kendall C, Stanbury E, Sendova-Franks AB, Franks NR. 2018 Complementary landmarks facilitate ant navigation. Behav. Processes 157, 702-710.

(doi:https://doi.org/10.1016/j.beproc.2018.03.004)

47. Lenoir A, Depickère S, Devers S, Christidès J-P, Detrain C. 2009 Hydrocarbons in the Ant Lasius niger: From the Cuticle to the Nest and Home Range Marking. J. Chem. Ecol. 35, 913-921.

(doi:10.1007/s10886-009-9669-6)

48. Wajnberg E, Acosta-Avalos D, Alves OC, De Oliveira JF, Srygley RB, Esquivel DMS. 2010

Magnetoreception in eusocial insects: An update. J. R. Soc. Interface 7, 207-225.

(doi:10.1098/rsif.2009.0526.focus)

49. Sandoval EL, Wajnberg E, Esquivel DMS, de Barros HL, Acosta-Avalos D. 2012 Magnetic Orientation in Solenopsis sp. Ants. J. Insect Behav. 25, 612-619. (doi:10.1007/s10905-012-9327-7)

50. Riveros AJ, Srygley RB. 2008 Do leafcutter ants, Atta colombica, orient their path-integrated home vector with a magnetic compass? Anim. Behav. 75, 1273-1281. (doi:10.1016/j.anbehav.2007.09.030)

51. Fleischmann PN, Grob R, Müller VL, Wehner R, Rössler W. 2018 The Geomagnetic Field Is a Compass Cue in Cataglyphis Ant Navigation. Curr. Biol. 28, 1440-1444.e2. (doi:10.1016/j.cub.2018.03.043)

52. Challet M, Fourcassié V, Blanco S, Fournier R, Theraulaz G, Jost C. 2005 A new test of random walks in heterogeneous environments. Naturwissenschaften 92, 367-370. (doi:10.1007/s00114-005-0001-1)

53. Wehner R, Srinivasan M V. 1981 Searching behaviour of desert ants, genus Cataglyphis (Formicidae, Hymenoptera). J. Comp. Physiol. $\square$ A 142, 315-338. (doi:10.1007/BF00605445)

54. Stuttard JP, Gottlieb D, Franks NR. 2016 Ants incommunicado: collective decision-making over new nest sites by ants with reduced communication. Behav. Ecol. Sociobiol. 70, 145-155.

(doi:10.1007/s00265-015-2033-7)

55. Franklin EL, Richardson TO, Sendova-Franks AB, Robinson EJH, Franks NR. 2011 Blinkered teaching: tandem running by visually impaired ants. Behav. Ecol. Sociobiol. 65, 569-579. (doi:10.1007/s00265010-1057-2)

56. Grüter C, Czaczkes TJ, Ratnieks FLW. 2011 Decision making in ant foragers (Lasius niger) facing conflicting private and social information. Behav. Ecol. Sociobiol. 65, 141-148. (doi:10.1007/s00265010-1020-2)

57. Czaczkes TJ, Grüter C, Jones SM, Ratnieks FLW. 2011 Synergy between social and private information increases foraging efficiency in ants. Biol. Lett. 7, 521-524. (doi:10.1098/rsbl.2011.0067)

58. Wang F, Landau DP. 2001 Efficient, Multiple-Range Random Walk Algorithm to Calculate the Density of States. Phys. Rev. Lett. 86, 2050-2053. (doi:10.1103/PhysRevLett.86.2050)

59. McNamara JM, Houston Al. 1987 Memory and the efficient use of information. J. Theor. Biol. 125, 385395. (doi:10.1016/S0022-5193(87)80209-6)

60. Britton NF, Stickland TR, Franks NR. 1998 Analysis of Ant Foraging Algorithms. J. Biol. Syst. 06, 315-336. (doi:10.1142/S0218339098000212)

61. Stickland TR, Britton NF, Franks NR. 1999 Models of information flow in ant foraging: the benefits of both attractive and repulsive signals. In Information Processing in Social Insects (eds C Detrain, JL Deneubourg, JM Pasteels), pp. 83-100. Basel: Birkhäuser Basel. (doi:10.1007/978-3-0348-8739-7_5)

62. Robinson EJH, Jackson DE, Holcombe M, Ratnieks FLW. 2005 Insect communication: 'No entry' signal in ant foraging. Nature 438, 442. (doi:10.1038/438442a)

63. Robinson EJH, Green KE, Jenner EA, Holcombe M, Ratnieks FLW. 2008 Decay rates of attractive and repellent pheromones in an ant foraging trail network. Insectes Soc. 55, 246-251.

(doi:10.1007/s00040-008-0994-5)

64. Robinson EJH, Ratnieks FLW, Holcombe M. 2008 An agent-based model to investigate the roles of attractive and repellent pheromones in ant decision making during foraging. J. Theor. Biol. 255, 250258. (doi:10.1016/j.jtbi.2008.08.015)

65. Fossum F, Montanier JM, Haddow PC. 2015 Repellent pheromones for effective swarm robot search in unknown environments. IEEE SSCI 2014 - 2014 IEEE Symp. Ser. Comput. Intell. - SIS 20142014 IEEE Symp. Swarm Intell. Proc. , 243-250. (doi:10.1109/SIS.2014.7011802)

66. Schroeder A, Ramakrishnan S, Kumar M, Trease B. 2017 Efficient spatial coverage by a robot swarm 
bioRxiv preprint doi: https://doi.org/10.1101/504241; this version posted May 28, 2020. The copyright holder for this preprint (which was not certified by peer review) is the author/funder. All rights reserved. No reuse allowed without permission.

based on an ant foraging model and the Lévy distribution. Springer US. (doi:10.1007/s11721-017-0132y)

67. Payton D, Daily M, Estowski R, Howard M, Lee C. 2001 Pheromone robotics. Auton. Robots 11, 319324. (doi:10.1023/A:1012411712038)

68. Van Dyke Parunak H, Brueckner SA, Sauter J. 2005 Digital Pheromones for Coordination of Unmanned Vehicles. In Environments for Multi-Agent Systems (eds D Weyns, H Van Dyke Parunak, F Michel), pp. 246-263. Berlin, Heidelberg: Springer Berlin Heidelberg.

69. Garnier S, Tâche F, Combe M, Grimal A, Theraulaz G. 2007 Alice in pheromone land: An experimental setup for the study of ant-like robots. Proc. 2007 IEEE Swarm Intell. Symp. SIS 2007, 37-44.

(doi:10.1109/SIS.2007.368024)

70. Arvin F, Krajnik T, Turgut AE, Yue S. 2015 COS $\Phi$ : Artificial pheromone system for robotic swarms research. IEEE Int. Conf. Intell. Robot. Syst. 2015-Decem, 407-412. (doi:10.1109/IROS.2015.7353405)

71. Hunt ER, Jones S, Hauert S. 2019 Testing the limits of pheromone stigmergy in high density robot swarms. R. Soc. Open Sci. 6, 190225. (doi:10.1098/rsos.190225)

72. Tinbergen N. 1963 On aims and methods of Ethology. Z. Tierpsychol. 20, 410-433. (doi:10.1111/j.14390310.1963.tb01161.x) 